\title{
Commentary: Mild endoplasmic reticulum stress ameliorates Ipopolysaccharide-induced neuroinflammation and cognitive impairment via regulation of microglial polarization
}

\author{
Sachchida N. Rai, Walia Zahra, Hareram Birla, Saumitra S. Singh and Surya P. Singh* \\ Department of Biochemistry, Institute of Science, Banaras Hindu University, Varanasi, India
}

Keywords: endoplasmic reticulum, lipopolysaccharide, neuroinflammation, cognitive impairment, microglia

\section{A commentary on}

OPEN ACCESS

Edited by:

Ashok Kumar

University of Florida, United States

Reviewed by:

Udai Pandey,

University of Pittsburgh Medical

Center, United States

Lakshmi Rajagopal,

Northwestern University, United States

Deep R. Sharma,

SUNY Downstate Medical Center,

United States

${ }^{*}$ Correspondence:

Surya P. Singh

suryasinghbhu16@gmail.com

Received: 29 December 2017

Accepted: 07 June 2018

Published: 25 June 2018

Citation:

Rai SN, Zahra W, Birla H, Singh SS and Singh SP (2018) Commentary:

Mild endoplasmic reticulum stress ameliorates

Ipopolysaccharide-induced neuroinflammation and cognitive impairment via regulation of microglial polarization

Front. Aging Neurosci. 10:192. doi: 10.3389/fnagi.2018.00192
Mild endoplasmic reticulum stress ameliorates lipopolysaccharide-induced neuroinflammation and cognitive impairment via regulation of microglial polarization by Wang, Y. W., Zhou, Q., Zhang, X., Qian, Q. Q., Xu, J. W., Ni, P. F., et al. (2017). J. Neuroinflammation 14:233. doi: 10.1186/s12974-017-1002-7

In this article, Wang and colleagues have discussed the non-harmful levels of Endoplasmic reticulum (ER) stress in rats focusing mainly on primary microglia. Specifically, they sought to investigate the regulation of lipopolysaccharide (LPS) driven neuroinflammation in male Sprague-Dawley rats through mild ER-stress (MERS). In experiment 1, to determine the extent of unfolded protein response (UPR), they measured expression of phosphorylated total protein kinase RNA-like ER kinase (p-PERK), phosphorylated eukaryotic translation initiation factor $2 \alpha$ (p-EIF2 $\alpha$ ), phosphorylated inositol-requiring protein $1 \alpha$ (p-IRE $1 \alpha)$, spliced X-box-binding protein-1 (XBP1s), XBP1u, activating transcription factor-4 (ATF4) and CCAAT/enhancer-binding protein homologous protein (CHOP) through western blot and Immunofluorescence (Wang et al., 2017). During ER stress, IRE1 $\alpha$ gets phosphorylated \& activated and cuts unspliced XBP1u mRNA into spliced XBP1s mRNA which further encodes XBP1 protein (Gardner et al., 2013). They found that expressions of $\mathrm{p}-\mathrm{IRE} 1 \alpha$ and XBP1s were considerably increased on administration of different doses of tunicamycin (TM), while the expression of XBP1u was significantly reduced. Expression of hippocampal p-PERK, p-EIF2 $\alpha$, ATF4, and CHOP were also assessed. p-PERK causes phosphorylation of EIF2 $\alpha$ (Walter and Ron, 2011; Hetz et al., 2015) which on prolonged phosphorylation induces paradoxical translation of ATF4 mRNA into its corresponding protein, in turn inducing upregulation of pro-apoptotic components such as CHOP (Gardner et al., 2013).

Furthermore, Caspase- 3 and cleaved caspase- 3 expressions were assessed in the CA1 region of the hippocampus. Increased expression of p-PERK and p-EIF2- $\alpha$ were seen at a range of different doses of TM administration, but ATF4, CHOP and cleaved caspase- 3 were only elevated at the highest dose of TM. Thus, the authors concluded that low doses of TM, i.e.; 0.3 and $3 \mu \mathrm{g} / 2$ $\mu \mathrm{l}$ in vivo \& 0.5 and $5 \mathrm{ng} / \mathrm{ml}$ in vitro led to modest UPR without cell or organism lethality as assessed by TUNEL labeling, while the higher concentrations of $30 \mu \mathrm{g} / 2 \mu \mathrm{l}$ in vivo and $50 \mathrm{ng} / \mathrm{ml}$ in vitro have shown serious ER perturbations and a robust UPR. In experiment 2, the authors studied the role of MERS in LPS-induced neuroinflammation and cognitive impairment 
in rats. MERS was induced by using $3 \mu \mathrm{g} / 2 \mu \mathrm{l} \mathrm{TM}$ and treated with or without sodium 4-phenylbutyrate (a stabilizing agent), an hour before the LPS administration both in vivo and in vitro. This low dose of TM $(3 \mu \mathrm{g})$ significantly improved freezing behavior and learning trials, indicating its role in protection against memory dysfunction caused by LPS.

They have also shown that TM prevented neurons from undergoing LPS-induced apoptosis. To clarify whether MERS was responsible for neuroprotective activity of TM, they administered rats with $100 \mathrm{mg} / \mathrm{kg}$ 4-Phenylbutyric acid (4-PBA) known to reduce ER stress, which at this dose does not affect normal functioning of nervous system. Treatment with 4-PBA significantly reduced expression levels of p-IRE1 $\alpha$ and XBP1s as compared to non-PBA treated groups. Also, neuroprotection conferred by TM was partially blocked by concomitant administration of 4-PBA as revealed by increased numbers of TUNEL-positive cells. Therefore, significant reductions in cognitive function in the TM+LPS+4-PBA group confirmed that low dose of TM protects against LPS-induced cognitive dysfunction by inducing MERS which inhibits caspase-3 activation (Wang et al., 2017). As in neurodegenerative disorders, microglia activation indicates an early sign of neuronal death, thus the authors tried to explore the effect of MERS on microglia in vitro by measuring expression of microglial genes associated with classic (M1), alternative repair and regeneration (M2a) and, immunomodulation (M2b) (Tang and Le, 2016). The relative expression of classical M1 genes CD86, CD32 and inducible nitric oxide synthase (iNOS), M2a genes YM1/2 and CD206 and, $\mathrm{M} 2 \mathrm{~b}$ gene suppressor of cytokine signaling 3 were assessed. LPS was shown to significantly increase mRNA levels of M1 and M2b markers in hippocampus compared with levels in the naïve group, while M2a genes were significantly reduced as compared to the naïve group. Alternatively, TM pretreatment led to alteration in balance of M1 and M2 microglia expression patterns in hippocampus significantly increasing expression of M2a genes and decreasing expression of M1 and M2b genes. Thus, the authors concluded that LPS-induced neuroinflammation was significantly attenuated by MERS, leading to a shift of the microglia population from $\mathrm{M} 1 / 2 \mathrm{~b}$ to $\mathrm{M} 2 \mathrm{a}$ in hippocampus. Further, the authors also used double immunofluorescent staining of iNOS and CD206 with microglial marker Iba1 in the hippocampal CA1 region, to show that TM inhibited

\section{REFERENCES}

Gardner, B. M., Pincus, D., Gotthardt, K., Gallagher, C. M., and Walter, P. (2013). Endoplasmicreticulum stress sensing in the unfolded protein response. Csh Perspect. Biol. 5:a13169. doi: 10.1101/cshperspect.a013169

Hetz, C., Chevet, E., and Oakes, S. A. (2015). Proteostasis control by the unfolded proteinresponse. Nat. Cell Biol. 17, 829-838. doi: 10.1038/ncb3184

Tang, Y., and Le, W. (2016). Differential roles of M1 and M2 microglia inneurodegenerative diseases. Mol. Neurobiol. 53, 1181-1194. doi: $10.1007 /$ s12035-014-9070-5

Walter, P., and Ron, D. (2011). The unfolded protein response: from stress pathway tohomeostatic regulation. Science 334, 1081-1086. doi: 10.1126/science.1209038

Wang, Y. W., Zhou, Q., Zhang, X., Qian, Q. Q., Xu, J. W., Ni, P. F., et al.. (2017). Mild endoplasmic reticulum stress ameliorates lipopolysaccharide-induced
LPS-induced microglia activation and shifted the phenotype of microglia toward M2a.

The authors also detected levels of TNF- $\alpha$, IL- $1 \beta$, and IL-6 proinflammatory factors thought to play major role in neuroinflammation. They have seen increased expression of these proinflammatory factors induced by LPS, while TM has significantly reduced this inflammatory response. But, the expression levels of these factors were significantly enhanced after 4-PBA co-treatment through reversal of anti-neuroinflammatory effects of TM. Results were further validated in vitro by demonstrating that dose of $5 \mathrm{ng} / \mathrm{ml}$ of TM was able to induce MERS in microglial culture. They have confirmed that TM has inhibited cytokine production and induced microglial polarization from $\mathrm{M} 1 / 2 \mathrm{~b}$ to $\mathrm{M} 2 \mathrm{a}$. Moreover, 4-PBA led to impairment of anti-inflammatory effects and M2a differentiation conferred by TM.

Since authors talk about neuroinflammation, they must have studied role of MERS on Astrogliosis. NF-kB plays important role in neuroinflammation, hence must have been investigated. The biochemical parameters such as Catalase and Lipid Peroxidation must have been checked in the hippocampal region. Moreover, on Page: 8 , in line "LPS significantly increased the mRNA levels of M1 and M2a markers in the hippocampus compared with the levels observed in the naïve group"; there should be M2b instead of $\mathrm{M} 2 \mathrm{a}$.

Thus, MERS has an important role in neuroinflammation and cognitive impairment. Since, ER stress has also been seen in astrocytes, an important cell type that plays a vital role in neurodegenerative disorders through neuroinflammation, the question arises whether MERS in astrocyte in LPS-induced neuroinflammation could also have contributed to the beneficial properties of MERS.

Finally, they reported that MERS preconditioning can alleviate neuroinflammation and cognitive impairment induced by LPS, thereby suggesting that moderate level of ER stress can act as a new therapeutic possibility to suspend or delay progression of neurodegenerative diseases (Wang et al., 2017).

\section{AUTHOR CONTRIBUTIONS}

All authors listed have made a substantial, direct and intellectual contribution to the work, and approved it for publication.

neuroinflammation and cognitive impairment via regulation of microglial polarization. J. Neuroinflammation 14:233. doi: 10.1186/s12974-017-1002-7

Conflict of Interest Statement: The authors declare that the research was conducted in the absence of any commercial or financial relationships that could be construed as a potential conflict of interest.

Copyright (C) 2018 Rai, Zahra, Birla, Singh and Singh. This is an open-access article distributed under the terms of the Creative Commons Attribution License (CC $B Y)$. The use, distribution or reproduction in other forums is permitted, provided the original author(s) and the copyright owner are credited and that the original publication in this journal is cited, in accordance with accepted academic practice. No use, distribution or reproduction is permitted which does not comply with these terms. 